\title{
RADON-NIKODYM PROPERTY IN SYMMETRIC SPACES OF MEASURABLE OPERATORS
}

\author{
XU QUANHUA
}

(Communicated by William J. Davis)

\begin{abstract}
Let $E$ be a rearrangement invariant function space on $(0, \infty)$ with the RNP. Let $(M, \tau)$ be a von Neumann algebra with a faithful normal semifinite trace $\tau$. It is proved that the associated symmetric space $L_{E}(M, \tau)$ of measurable operators has the RNP.
\end{abstract}

\section{INTRODUCTION}

The aim of this note is to solve a problem arising in a previous work [10] concerning the Radon-Nikodym property (RNP) in symmetric spaces of measurable operators. For stating our result, we introduce the necessary definitions and notations (for unexplained notions, see $[5,6,9]$ ). We also refer the reader to [2] for the definition and the elementary properties of the RNP.

Let $(M, \tau)$ be a semifinite von Neumann algebra acting on a Hilbert space $H$, with a faithful normal semifinite trace $\tau$. Let $\bar{M}$ be the space of all measurable operators with respect to $(M, \tau)$ in the sense of [7], equipped with the measure topology defined there. For $a \in \bar{M}$ and $t>0$ the $t$ th $s$-number (singular number) of $a$ is defined by (cf. [3])

$$
\mu_{t}(a)=\inf \{\|a e\|: e \text { is a projection in } M \text { with } \tau(1-e) \leq t\} .
$$

The function $t \rightarrow \mu_{t}(a)$ on $(0, \infty)$ is denoted by $\mu(a)$. This is a positive nonincreasing function on $(0, \infty)$. Note that $\mu(a)=\mu\left(a^{*}\right)=\mu(|a|),|a|$ being the absolute value of $a$. Recall the following useful formula for $\mu_{t}(a)$ in terms of the spectral measures (cf. [3])

$$
\mu_{t}(a)=\inf \left\{s \geq 0 \mid \lambda_{s}(a) \leq t\right\},
$$

where $\lambda_{s}(a)=\tau\left(e_{(s, \infty)}(|a|)\right), e_{(s, \infty)}(|a|)$ being the spectral projection of $|a|$ corresponding to the interval $(s, \infty)$ for $s \geq 0$. It follows immediately that

$$
\mu_{t}(a)=0 \text { for } t \geq \tau(\operatorname{supp}(a)),
$$

where $\operatorname{supp}(a)$, the support of $a$, is the smallest projection $e$ in $M$ such that $a e=a$. In particular, if $\tau$ is finite, i.e., $\tau(1)<\infty$, then for any $a \in \bar{M}$

$$
\mu_{t}(a)=0 \text { for } t \geq \tau(1) \text {. }
$$

Received by the editors April 13, 1990 and, in revised form, December 4, 1990.

1980 Mathematics Subject Classification (1985 Revision). Primary 46B22, 46C05, 46L50.

Key words and phrases. Radon-Nikodym property, symmetric space, semifinite von Neumann algebra, measurable operator. 
Now let $E$ be a rearrangement invariant (r.i.) function space on $(0, \infty)$ in the sense of [6]. Then we define the symmetric space of measurable operators associated with $E$ and $(M, \tau)$ as follows:

$$
L_{E}(M, \tau)=\{a \in \bar{M} \mid \mu(a) \in E\}
$$

and

$$
\|a\|_{L_{E}(M, \tau)}=\|a\|_{E}=\|\mu(a)\|_{E}, \quad a \in L_{E}(M, \tau) .
$$

It is an elementary fact that $\left(L_{E}(M, \tau),\|\cdot\|\right)$ is a Banach space (cf. [10, Lemma 4.1]). Moreover, we have $\|a\|_{E}=\left\|a^{*}\right\|=\||a|\|_{E}$ for any $a \in L_{E}(M, \tau)$. If $E=L_{p}(0, \infty) \quad(0<p \leq \infty)$, then $L_{E}(M, \tau)$, denoted usually by $L_{p}(M, \tau)$ in this case, is simply the usual noncommutative $L_{p}$-space associated with a semifinite von Neumann algebra. Note that $L_{\infty}(M, \tau)$ is just $M$ equipped with the operator norm.

If $\tau$ is finite, by (2) we have $\mu_{t}(a)=0$ for $t \in[\tau(1), \infty) \quad(a \in \bar{M})$; so that in this case $E$ can be taken as an r.i. function space on $(0, \tau(1))$.

It would be natural to expect properties of a symmetric space of measurable operators to reflect the properties of the corresponding r.i. function space. Thus we proved in [11] that uniform convexity and uniform PL-convexity pass from $E$ to $L_{E}(M, \tau)$, and in [10] that the same phenomenon occurs for the analytic Radon-Nikodym property and the uniform $H$-convexity in the sense of [12]. A related problem arising from [10] is to know whether the RNP also passes from $E$ to $L_{E}(M, \tau)$. We answer this question by the following result.

Theorem. Let $E$ be an r.i. function space on $(0, \infty)$ having the RNP. Then for any semifinite von Neumann algebra $(M, \tau), L_{E}(M, \tau)$ possesses the $R N P$.

It is worthwhile to note that a similar result for unitary ideals is easy (of course, our theorem contains this special case). In that case, $E$ is a symmetric Banach sequence space on $\mathbf{N}$ and $(M, \tau)$ is just $(B(H), \operatorname{tr})$, where $B(H)$ is the space of all bounded operators on a separable Hilbert space $H$ and $\operatorname{tr}$ is the usual trace on $B(H)$. Traditionally, $L_{E}\left(B(H)\right.$, tr) is denoted by $C_{E}$, the unitary ideal associated with $E$. If $E$ has the RNP, $E$ fails to contain $c_{0}$. Then it follows easily that the canonical basis is a boundedly complete basis for $E$ with a basis constant 1 . Therefore $E$ is order isometric to the dual of $E^{\prime}, E^{\prime}$ being the closed subspace of the dual $E^{*}$ of $E$ generated by all finite sequences (cf. [5]). Now by a well-known result from the theory of unitary ideals (cf. [4, Theorem III.12.2]), we have

$$
\left(C_{E^{\prime}}\right)^{*}=C_{\left(E^{\prime}\right)^{*}}=C_{E} .
$$

Note also that $C_{E}$ is separable because $E$ is separable. Hence $C_{E}$ is a separable dual, so it has the RNP. This simple reasoning also shows that for symmetric Banach sequence spaces and unitary ideals, the RNP is equivalent to the absence of $c_{0}$ in these spaces. Therefore in these spaces the RNP and the analytic RNP coincide.

For further results about symmetric spaces of measurable operators the reader is referred to [10].

\section{Proof}

Now we proceed to prove the theorem. Our proof relies heavily on the idea in the proof of the corresponding result for the analytic RNP in [10]. In the 
following, $E$ always denotes an r.i. function space on $(0, \infty)$ and $(M, \tau)$, a semifinite von Neumann algebra on $H$, with a faithful normal semifinite trace $\tau$.

As in [10], we first consider the finite trace case.

Lemma 1. Suppose $\tau(1)=1$. Let $F$ be an order continuous r.i. function space on $(0,1)$. Then

$$
\left(L_{F}(M, \tau)\right)^{*}=L_{F^{*}}(M, \tau) .
$$

Proof. Note first that the order continuity of $F$ implies that $F^{*}$ consists only of measurable functions on $(0,1)$ (cf. [6]). Hence $F^{*}$ is also an r.i. function space on $(0,1)$, so $L_{F^{*}}(M, \tau)$ is well defined.

The inclusion

$$
L_{F^{*}}(M, \tau) \subset\left(L_{F}(M, \tau)\right)^{*} \quad(\text { of norm } \leq 1)
$$

is easily seen. Indeed, by [3, Theorem 4.2], for any $b \in L_{F^{*}}(M, \tau)$ and any $a \in L_{F}(M, \tau)$, we have

$$
\int_{0}^{1} \mu_{t}(a b) d t \leq \int_{0}^{1} \mu_{t}(a) \mu_{t}(b) d t .
$$

Thus $\tau(a b)$ is well defined and

$$
|\tau(a b)| \leq \int_{0}^{1} \mu_{t}(a) \mu_{t}(b) d t .
$$

It follows that the linear functional $l: a \mapsto \tau(a b)$ defined on $L_{F}(M, \tau)$ is continuous and of norm $\leq\|\mu(b)\|_{F^{*}}=\|b\|_{F^{*}}$.

For the converse inclusion we take $l \in\left(L_{F}(M, \tau)\right)^{*}$. Then we must show that $l$ is defined by an element $b \in L_{F^{*}}(M, \tau)$ as above and that the norm of $l$ in $\left(L_{F}(M, \tau)\right)^{*}$ is less or equal to $\|b\|_{L_{F^{*}}(M, \tau)}$. For this we first show that $l$ is in the predual of $M$, that is, $l$ is an element of $L_{1}(M, \tau)$ (it is well known that $\left.\left(L_{1}(M, \tau)\right)^{*}=L_{\infty}(M, \tau)=M\right)$.

Because $F$ is an r.i. function space on $(0,1)$, we have (cf. [6])

$$
\left.L_{\infty}(0,1) \subset F \subset L_{1}(0,1) \quad \text { (inclusions of norm } \leq 1\right) .
$$

It follows that

$$
M \subset L_{F}(M, \tau) \subset L_{1}(M, \tau) \text { (inclusions of norm } \leq 1 \text { ). }
$$

Therefore the continuous linear functional $l$ on $L_{F}(M, \tau)$ also defined a continuous linear functional on $M$, that is, $l \in M^{*}$. In order to show that $l$ is in the predual of $M$, by a well-known result from the theory of operator algebras (cf. [9, Corollary III.3.11]), it suffices to show the following: For any orthogonal family $\left\{e_{i}\right\}_{i \in I}$ of projections in $M$

$$
l\left(\sum_{i \in I} e_{i}\right)=\sum_{i \in I} l\left(e_{i}\right)
$$

Equation (3) immediately follows from the following lemma. 
Lemma 2. Let $\left\{e_{i}\right\}_{i \in I}$ be an orthogonal family of projections of $M$. Then $\sum_{i \in I} e_{i}$ converges in $L_{F}(M, \tau)$.

Proof. For each finite subset $J$ of $I$, let $h_{J}=\sum_{i \in J} e_{i}$, and let $k_{J}=\sum_{i \notin J} e_{i}$ (convergence in the strong operator topology). Then the decreasing net $\left\{k_{J}: J\right.$ finite subset of $I\}$ converges to 0 in the strong operator topology. Since $\tau$ is normal and finite, the net $\left\{\tau\left(k_{J}\right)\right\}$ converges to 0 . Since $F$ is order continuous, $\left\{\left\|\chi_{\left[0, \tau\left(k_{J}\right)\right]}\right\|_{F}\right\} \rightarrow 0, \chi_{w}$ being the indicator function of a subset $w$. As $\mu\left(k_{J}\right)=$ $\chi_{\left[0, \tau\left(k_{J}\right)\right]},\left\{k_{J}\right\} \rightarrow 0$ in $L_{F}(M, \tau)$. This proves Lemma 2.

End of the proof of Lemma 1 . Now $l$ is in the predual of $M$; namely, there exists a measurable operator $b \in L_{1}(M, \tau)$ such that

$$
l(a)=\tau(a b), \quad \forall a \in M .
$$

Consequently

$$
|\tau(a b)| \leq\|l\|\|a\|_{F}, \quad \forall a \in M .
$$

Now by [10, Lemma 4.5], $M$ is dense in $L_{F}(M, \tau)$; therefore, for any $a \in$ $L_{F}(M, \tau), \tau(a b)$ is well defined and (4) and (5) hold for $a \in L_{F}(M, \tau)$. We next show that $b \in L_{F^{*}}(M, \tau)$ and $\|b\|_{F^{*}} \leq\|l\|$.

Let $b=u|b|$ be the polar decomposition of $b$ and $|b|=\int_{0}^{\infty} t d e_{t}$ be the spectral decomposition of $|b|$. Let $\tilde{e}_{t}=e_{\mu_{t}(b)-0}\left(t>0, e_{0-0}=1\right)$. Then $|b|$ admits the following Schmidt decomposition (cf. [8])

$$
|b|=\int_{0}^{1} \mu_{t}(b) d \tilde{e}_{t}
$$

Let $x$ be a nonincreasing positive function in $F$ such that $x$ is constant in the intervals where $\mu(b)$ is constant. Define

$$
a=\left(\int_{0}^{1} x(t) d \tilde{e}_{t}\right) \cdot u^{*}
$$

Then it is easy to check that $a \in L_{F}(M, \tau)$ and $\|a\|_{F} \leq\|x\|_{F}$. We also have

$$
\int_{0}^{1} x(t) \mu_{t}(b) d t=\tau\left(\int_{0}^{1} x(t) d \tilde{e}_{t} \cdot \int_{0}^{1} \mu_{t}(b) d \tilde{e}_{t}\right)=\tau(a b) .
$$

Then by $(5)$

$$
\int_{0}^{1} x(t) \mu_{t}(b) d t \leq\|l\|\|a\|_{F} \leq\|l\|\|x\|_{F} .
$$

Taking the supremum in the above inequalities over all $x$ satisfying the previous property and $\|x\|_{F} \leq 1$, we deduce that $\mu(b) \in F^{*}$ and $\|\mu(b)\|_{F^{*}} \leq\|l\|$. Consequently, $b \in L_{F^{*}}(M, \tau)$ and $\|b\|_{L_{F^{*}}(M, \tau)} \leq\|l\|$. This concludes the proof of Lemma 1.

Lemma 3. Assume that $\tau$ is finite and $E$ has the RNP. Then $L_{E}(M, \tau)$ has the RNP.

Proof. After a trivial normalization, we can assume $\tau(1)=1$. Then we can regard $E$ as an r.i. function space on $(0,1)$. The RNP of $E$ implies that $E$ is not isomorphic to $L_{1}(0,1)$ and that $E$ is maximal in the sense of [6]. 
Consequently, $E=F^{*}$, where $F$ is an order continuous r.i. function space on $(0,1)$ (cf. [6]). Then by Lemma 1

$$
L_{E}(M, \tau)=\left(L_{F}(M, \tau)\right)^{*} .
$$

If $L_{E}(M, \tau)$ was separable, then $L_{E}(M, \tau)$, as a separable dual, would have the RNP. But unfortunately, $L_{E}(M, \tau)$ is in general nonseparable. Therefore, we must do something else in order to prove the RNP of $L_{E}(M, \tau)$.

Now let $X$ be a separable closed subspace of $L_{E}(M, \tau)$. We show that $X$ is isometric to a closed subspace of a separable dual, from which [2, Corollary III.3.5] and Lemma 3 follows.

Let $\left\{a_{n}\right\}_{n>0}$ be a dense sequence in $X$; by [10, Lemma 4.3], $M$ is dense in $L_{E}(M, \tau)$. Then by approximating $a_{n}$ by elements in $M$, we may assume $a_{n} \in M$ for any $n \geq 0$. Now let $\widetilde{M}$ be the von Neumann subalgebra of $M$ generated by 1 and all the $a_{n}$ 's. Let $\tilde{\tau}$ be the restriction of $\tau$ to $\widetilde{M}$. Clearly, $\tilde{\tau}$ is a faithful normal finite trace on $\widetilde{M}$. It is also clear that $L_{E}(\widetilde{M}, \tilde{\tau})$ is naturally identified with a closed subspace of $L_{E}(M, \tau)$ and $X$ a closed subspace of $L_{E}(\widetilde{M}, \tilde{\tau})$. Now by Lemma $1, L_{E}(\widetilde{M}, \tilde{\tau})$ is a dual. Furthermore, by [10, Lemma 5.6], $L_{E}(\widetilde{M}, \tilde{\tau})$ is separable. Thus, $X$ is a closed subspace of the separable dual $L_{E}(\widetilde{M}, \tilde{\tau})$, proving Lemma 3 .

Lemma 3 proves the theorem in the finite trace case. We reduce the general case to the finite trace case by using the semifiniteness of $\tau$. The following argument is similar to the corresponding part in the proof of Theorem 5.1 in [10]. We outline it only. In the following, $E$ is an r.i. function space on $(0, \infty)$ with the RNP.

We use the following characterization of the RNP due to Bukhvalov and Danilevich [1]. Let $X$ be a Banach space. Let $h_{\infty}(X)$ denote the space of all bounded harmonic $X$-values functions in the unit disc of the complex plane. Then $X$ has the RNP iff every function $f \in h_{\infty}(X)$ admits almost everywhere radial limits in $X$ on the unit circle $\mathbf{T}$, that is, $\lim _{r \rightarrow 1} f\left(r e^{i \theta}\right)$ exists in $X$ almost everywhere on $\mathbf{T}$.

Let $f \in h_{\infty}\left(L_{E}(M, \tau)\right)$. We show that $f$ admit almost everywhere radial limits in $L_{E}(M, \tau)$ on $\mathbf{T}$, from which the theorem follows. Write

$$
f\left(r e^{i \theta}\right)=\sum_{n \in \mathbf{Z}} a_{n} r^{|n|} e^{i n \theta}, \quad 0 \leq r<1,0 \leq \theta \leq 2 \pi,
$$

where $a_{n} \in L_{E}(M, \tau) \quad(n \in \mathbf{Z})$ and $\lim \sup _{n \rightarrow \pm \infty}\left\|a_{n}\right\|_{E}^{1 / n} \leq 1$. By the semifiniteness of $\tau$ we find an orthogonal family $\left\{e_{i}\right\}_{i \in I}$ of projections in $M$ such that $\tau\left(e_{i}\right)<\infty$ for every $i \in I$ and such that

$$
1=\sum_{i \in I} e_{i} \quad \text { (convergence in the strong operator topology). }
$$

By [10, Lemma 5.7], for every $n \in \mathbf{Z},\left\{i \in I:\left\|a_{n} e_{i}\right\|_{E} \neq 0\right.$ or $\left.\left\|e_{i} a_{n}\right\|_{E} \neq 0\right\}$ is at most countable; so that there exists an at most countable subset $\left\{e_{k}\right\}_{k \geq 0}$ of $\left\{e_{i}\right\}_{i \in I}$ such that $\left\|e_{k} a_{n}\right\|_{E} \neq 0$ or $\left\|a_{n} e_{k}\right\|_{E} \neq 0$ for some $n \in \mathbf{Z}$. Let $e=\sum_{k \geq 0} e_{k}$. Then we have $e f(z)=f(z) e=f(z) \quad(z \in D)$. Therefore replacing $M$ by $e M e$ and $\tau$ by its restriction to $e M e$, we can assume $e=1$. For $j \geq 0, k \geq 0$, set $e_{j k}=e_{j} \vee e_{k}$ (maximum taken in the lattice of all the 
projections in $M$ ). Now let $M_{e_{j k}}=e_{j k} M e_{j k}$ and $\tau_{e_{j k}}$ be the resiriction of $\tau$ to $M_{e_{j k}}(j \geq 0, k \geq 0)$. $\tau_{e_{j k}}$ is a finite trace on $M_{e_{j k}}$. For $j \geq 0, k \geq 0$ consider $f_{j k}(z)=e_{j} f(z) e_{k} \quad(z \in D)$. Regarded as a function with values in $L_{E}\left(M_{e_{j k}}, \tau_{e_{j k}}\right), f_{j k} \in h_{\infty}\left(L_{E}\left(M_{e_{j k}}, \tau_{e_{j k}}\right)\right)$ and

$$
\left\|f_{j k}(z)\right\|_{L_{E}\left(M_{e_{j k}}, \tau_{e_{j k}}\right)} \leq\|f(z)\|_{L_{E}(M, \tau)}, \quad z \in D .
$$

By Lemma 3, $L_{E}\left(M_{e_{j k}}, \tau_{e_{j k}}\right)$ has the RNP; so in $L_{E}\left(M_{e_{j k}}, \tau_{e_{j k}}\right)$

$$
\lim _{r \rightarrow 1} f_{j k}\left(r e^{i \theta}\right)=\varphi_{j k}\left(e^{i \theta}\right) \quad \text { almost everywhere on } \mathbf{T} \text {. }
$$

We can evidently extend the boundary function $\varphi_{j k}$ to a function with values in $L_{E}(M, \tau)$. This new function is still denoted by $\varphi_{j k}$. Then it satisfies

$$
e_{j} \varphi_{j k}\left(e^{i \theta}\right)=\varphi_{j k}\left(e^{i \theta}\right) e_{k}=\varphi_{j k}\left(e^{i \theta}\right) \text { almost everywhere on } \mathbf{T} ;
$$

furthermore, the above almost everywhere radial limits also exist in $L_{E}(M, \tau)$. Then by [10, Lemma 5.7], we can show that $\sum_{j \geq 0} \sum_{k \geq 0} \varphi_{j k}\left(e^{i \theta}\right)$ converges in $L_{E}(M, \tau)$ almost everywhere on $\mathbf{T}$ (cf. [10] for more details). Let

$$
\varphi\left(e^{i \theta}\right)=\sum_{j \geq 0} \sum_{k \geq 0} \varphi_{j k}\left(e^{i \theta}\right), \quad 0 \leq \theta \leq 2 \pi .
$$

Then $\varphi$ is a bounded measurable function on $\mathrm{T}$ with values in $L_{E}(M, \tau)$ since for any $m \geq 0, n \geq 0$

$$
\begin{aligned}
\operatorname{ess} \sup _{\theta \in \mathbf{T}}\left\|\sum_{j=0}^{m} \sum_{k=0}^{n} \varphi_{j k}\left(e^{i \theta}\right)\right\|_{L_{E}(M, \tau)} & \leq \sup _{z \in D}\left\|\sum_{j=0}^{m} \sum_{k=0}^{n} f_{j k}(z)\right\|_{L_{E}(M, \tau)} \\
& \leq \sup _{z \in D}\|f(z)\|_{L_{E}(M, \tau)} .
\end{aligned}
$$

Let

$$
F\left(r e^{i \theta}\right)=\int_{0}^{2 \pi} \varphi\left(e^{i \eta}\right) P_{r}(\theta-\eta) \frac{d \eta}{2 \pi} \quad(0 \leq r<1,0 \leq \theta \leq 2 \pi)
$$

be the Poisson integral of $\varphi$ in the unit disc, $P_{r}$ being the Poisson kernel. By the dominated convergence theorem

$$
F(z)=\sum_{j \geq 0} \sum_{k \geq 0} F_{j k}(z), \quad z \in D,
$$

where $F_{j k}$ is the Poisson integral of $\varphi_{j k}(j \geq 0, k \geq 0)$. Since $\varphi_{j k}$ is the almost everywhere radial limit of $f_{j k}, F_{j k}=f_{j k}$; so that

$$
F(z)=\sum_{j \geq 0} \sum_{k \geq 0} f_{j k}(z), \quad z \in D .
$$

On the other hand, it is clear that

$$
f(z)=\sum_{j \geq 0} \sum_{k \geq 0} f_{j k}(z), \quad z \in D .
$$


Consequently, $F=f$. Hence $f$ is the Poisson integral of $\varphi$. Therefore

$$
\lim _{r \rightarrow 1} f\left(r e^{i \theta}\right)=\varphi\left(e^{i \theta}\right) \quad \text { in } L_{E}(M, \tau) \text { almost everywhere on } \mathbf{T},
$$

which completes the proof of the theorem.

\section{ACKNOWLEDGMENTS}

The author would like to thank the referee for several suggestions, especially for pointing out the present simple proof of Lemma 2, which replaces an originally longer one.

\section{REFERENCES}

1. A. V. Bukhvalov and A. A. Danilevich, Boundary properties of analytic and harmonic functions with values in Banach spaces, Math. Notes 31 (1982), 104-110.

2. J. Diestel and J. J. Uhl, Vector measures, Amer. Math. Soc., Providence, RI, 1977.

3. T. Fack and H. Kosaki, Generalized s-numbers of $\tau$-measurable operators, Pacific J. Math. 123 (1986), 269-300.

4. I. C. Gohberg and M. G. Krein, Introduction to the theory of linear non self-adjoint operators, Transl. Math. Monographs, vol. 18, Amer. Math. Soc., Providence, RI, 1969.

5. J. Lindenstrauss and L. Tzafriri, Classical Banach spaces I, Springer-Verlag, Berlin, Heidelberg, and New York, 1977.

6. __ Classical Banach spaces II, Springer-Verlag, Berlin, Heidelberg, and New York, 1979.

7. E. Nelson, Notes on non-commutative integration, J. Funct. Anal. 15 (1974), 103-116.

8. V. I. Ovchinnikov, s-numbers of measurable operators, Funct. Anal. Appl. 4 (1970), 236242.

9. M. Takesaki, Theory of operator algebras, Springer-Verlag, Berlin, Heidelberg, and New York, 1979.

10. Q. Xu, Analytic functions with values in lattices and symmetric spaces of measurable operators, Math. Proc. Camb. Phil. Soc. 109 (1991), 541-563.

11. __ Convexité uniforme des espaces symétriques d'opérateurs measurables, C.R. Acad. Sci. Paris Sér. I. Math. 309 (1989), 251-254.

12. __ Convexités uniformes et inégalités de martingales, Math. Ann. 287 (1990), 193-211.

Université des Sciences et Techniques de Lille Flandres Artois, U.F.R. De Mathématiques Pures et AppliQuées, U.R.A. C.N.R.S. 751, 59655 Villeneuve d’AscQ Cedex France 